Volume 1, Issue 2 | Pages 60-65 | ISSN - Applied

Received: 25 Apr 2019| Accepted: 26 Apr 2019| Published: 29 Apr 2019

\title{
SSL: SMART STREET LAMP BASED ON FOG COMPUTING FOR SMARTER CITIES
}

\author{
N. NITHYA ${ }^{*}$, S. BHUVANESHWARI ${ }^{2}$ \& S. ARIVUKODI ${ }^{2}$ \\ ${ }^{1}$ Assistant Professor, Department of Computer Applications, Dhanalakshmi Srinivasan College of Arts and Science for \\ Women (Autonomous), Bharathidhasan University, Perambalur, Tamil Nadu, India \\ ${ }^{2}$ Research Scholar, Department of Computer Applications, Dhanalakshmi Srinivasan College of Arts and Science for \\ Women (Autonomous), Bharathidhasan University, Perambalur, Tamil Nadu, India \\ *Corresponding Author: N. Nithya (MCA, MPhil), Assistant Professor, Department of Computer Applications, \\ Dhanalakshmi Srinivasan College of Arts and Science for Women (Autonomous), Bharathidhasan University, \\ Perambalur, Tamil Nadu, India
}

\section{$\underline{\text { ABSTRACT }}$}

Energy conservation and higher performance could be a should in sensible street lamps assembled in smart cities. the prevailing street lights don't seem to be appropriate for sensible cities wherever the upkeep and security is quiet sophisticated. so as to deal with these issues, a sensible streetlight (SSL) is planned in our idea. each sensible lamp may be operated severally which ends in an exceedingly fine management. all told sensible street lamps brightness may be adjusted dynamically. every streetlight will report the abnormal standing severally with an automatic alarm. The experimental results showed that planned SSL will improve energy potency and cut back danger. Intelligent street lamps are planned supported several communication technologies, like ZigBee, LPWA, GSM, and so on . moreover, there are several alternative communication technologies, like Bluetooth, UMTS/LTE, Wi-Fi, and so on. every of those communication technologies has its own characteristic. the road lamp is provided with some sensors, like location detector, infrared detector, and light-weight detector, to make associate degree intelligent sensing streetlight. Consequently, brightness of street lamps may be adjusted. As already mentioned, safety is one in all the foremost necessary characteristics of the sensible cities, and periodic maintenance of street lamps could be a key parameter for safety. The shorter the upkeep is, the higher the security is going to be.

KEYWORDS: sensible streetlight, SSL, sensible lamp, communication technologies, street lamps, safety

\section{INTRODUCTION}

\section{Project DesCRiption}

Both safety and energy conservation are vital benefits of sensible cities. Namely, town streetlight is related to with each safety and energy conservation. Therefore, streetlight is an important a part of the sensible cities. However, current street lamps have lack of sensible characteristics, that will increase each danger and energy consumption. so as to deal with these issues, a sensible streetlight (SSL) supported fog computing for smarter cities is projected during this paper. the benefits of the projected SSL are:

1. fine management, as a result of each streetlight will be operated independently; 
2. dynamic brightness adjustment, all street lamps will be adjusted dynamically;

3. autonomous alarm on abnormal states, every streetlight will report the abnormal standing severally, like broken, stolen, and so on.

The experimental results showed that projected SSL will improve energy potency and scale back danger.

\section{Problem Definition}

The major cause in the usual street lamps are the lamps may be easily stolen, breakable and can be damaged in day to day life cycle. Moreover the maintenance of the street lamps are not up to the core. To validate those major deficiency causes the street lamps are in urge to make an change over.

In the current environment the stability and the durability of every lamps must be higher, but to do so the normal procedure has not been supported to make that process to work with efficiency.

\section{LITERATURE SURVEY}

\section{A Measurement Study OF Vehicular Internet ACCeSS \\ Authors: Vladimir Bychkovsky, Bret Hull, Allen Miu, Hari Balakrishnan, Samuel Madden}

Internet services are going to be pervasive in future intelligent transportation systems. to the current finish, conveyance Wi-Fi networks area unit introduced and wide deployed these days. However, quantitative performance in conveyance Wi-Fi networks has not been reported within the literature. during this paper, we have a tendency to conduct a measuring study in conveyance Wi-Fi networks to research the transmission and downlink output below numerous conveyance environments. The measuring results demonstrate that current conveyance Wi-Fi networks have poor quality in terms of downlink output and therefore economical resource management schemes ought to be devised.

\section{WIRELESS NETWORKS AND TRANSCEIVERS}

\section{Authors: Farahani. ZigBee}

In his article describes the planning issues for low-power short-range radio transceivers with a spotlight on the two.4 GHz PHY layer outlined as a part of the IEEE 802.15.4 standard. The specification needs for IEEE 802.15.4compliant transceivers, and therefore the style challenges and sensible implementation of a highly-integrated low-power a pair of.4 $\mathrm{GHz}$ transceiver ar afterwards mentioned. The transceiver uses a direct-conversion receiver with switched antenna diversity and a transmitter mistreatment direct closedloop VCO modulation. It integrates a mask-programmable radio controller capable of autonomously playing timing-critical macintosh functions, and a sleep timer. enforced in zero.18 mum RFCMOS technology with a chip space of but six mm2, the transceiver achieves a link margin of ninety nine $\mathrm{dB}$ whereas drawing sixteen. $8 \mathrm{~mA}$ and eighteen $\mathrm{mA}$ from a one. $8 \mathrm{~V}$ offer in receive and transmit mode, severally.

\section{Calculation of the Spatial Preprocessing and link Adaption feedback for 3GPP UMTS/LTE.WIRELESS ADVANCED Authors: S Schwarz, C Mehlfuhrer, M Rupp}

This paper presents associate economical methodology for scheming the Precoding Matrix Indicator (PMI), Rank Indicator (RI) and Channel Quality Indicator (CQI) at an extended Term Evolution (LTE) User instrumentation (UE). the indications ar needed for abstraction preprocessing and link adaption within the downlink of a 3GPP 
UMTS/LTE system. to scale back the process burden for the UE, our methodology decomposes the matter into 2 separate steps, one in all together evaluating the PMI and American state supported a mutual info metric and one in all selecting the CQI worth to realize a given target Block Error magnitude relation (BLER) constraint. The performance of the tactic is evaluated utilizing associate LTE downlink physical layer machine. The influence of calculable channel information on the feedback alternative is investigated for statistical procedure (LS) and Linear Minimum Mean square Error (LMMSE) channel estimators.

\section{SYSTEM DESIGN}

To propose a smart street lamp (SSL) based on fog computing for smarter cities to meet the above four abilities. The proposed SSL consists of three main parts: an intelligent sensing street lamp, which can adjust lamp brightness, an autonomous alarm which reports about abnormal behavior; an efficient network, which is used for real-time communication between managers and massive street lamps; and lastly, a flexible management platform, which is easy and highly automated. We verified the proposed SSL by its application in Xiasha District of Hangzhou, China, and very good results were obtained. The average maintenance period, which denotes the time period from the moment the street lamp is broken to the moment that is noticed by the server, is less than 20 minutes. Moreover, the proposed SSL can reduce human resources avoiding an inefficient manual patrol. The main contributions of the proposed SSL are: The hybrid network is adopted, the Narrow Band Internet of Things (NB-IoT) is used for real-time communication between server and massive street lamps, and the Internet is used for real-time communication between managers and server; A flexible management platform is implemented, and it notifies the managers about broken street lamps at real time and automatically dispatches the maintenance staff to repair broken street lamps. The states of all street lamps can be traced and adjusted in real time.



\section{MODULE DESCRIPTIONS}

\section{ADMIN}

The admin controls the entire system through online in which the whole smart street lights has been covered. The admin has the proper authentication to operate the total number of street lights that has been involved in the system.

\section{UNIQUE ID GENERATION}

After the process has been undertaken by the admin each smart street light has been provided with an unique ID from which the admin can easily identify the exact smart street lamp for further operations like ON/OFF, maintenance, 
stolen, broken etc. By assuming the unique ID every smart lamp can be handled under the control of the admin.

\section{MONitoring ChaRT}

We evaluate the effectiveness of the proposed SSL by simulation of abnormal states of street lamps based on fog computing server which implements flexible management platform. We analyze the periodic maintenance of abnormal states of street lamps. The results of SSL reliability; and finally, the energy conservation has been solved.

\section{CONTROL ROOM SURVEILLANCE}

The server will check the abnormal states, the server will automatically send the information on abnormal lamp state consisting of street lamp ID, lamp location, and abnormal state description to the managers and nearest serviceman. Therefore, the maintenance period can be decreased seriously. The managers can send commands using mobile application or Web browser. Therefore, the serviceman can receive the tasks through mobile application or SMS.

\section{STREET LIGHT INFORMATION}

The Smart Street Light SSL consists of three main parts: an intelligent sensing street lamp, which can adjust lamp brightness, an autonomous alarm which reports about abnormal behavior; an efficient network, which is used for real-time communication between managers and massive street lamps; and lastly, a flexible management platform, which is easy and highly automated.

\section{Receiving Street Light Status}

Using both the intelligent sensing street lamps and efficient network, the server gets the information of all street lamps, consisting of lamps states, locations, external environment brightness, and so on. Therefore, the management platform is a key factor of the street lamp management system. Here, we implement a flexible management platform based on fog computing, which simplifies the management system. Moreover, the fog computing based server offers better real time response, while cloud computing delivers the elastic computing power and storage at a lower cost.

As already mentioned, all street lamps periodically send information of their states to the server, and the server stores received information in the database. In order to find the abnormal states of street lamps, the server periodically checks.

\section{Monitoring Power Details}

The check cycle can be set by managers. The longer the check cycle is, the longer the maintenance cycle of a broken street lamp is, and the lower the system cost is. Contrary, the shorter the check cycle is, the shorter the maintenance cycle of a broken street lamp is, and the higher the system cost is. 20 minute can be tolerable for both maintenance cycle and system cost. Therefore, to balance these two sides, we set the check cycle to 20 minutes, i.e. server checks states in database every 20 minutes.

\section{SMART LAMP MODULE}

\section{Current Status of SMart Lamp}

\section{- Check Status (ON/OFF)}

The states of all street lamps can be traced and adjusted in real time The street lamp periodically send reports on its current and voltage values. Based on the current and voltage values of the street lamp, the server can determine the street lamp state. If the current of the street lamp is zero, but the voltage is not zero, then the server 
can conclude that the light bulb may be broken.

\section{- Intimate Status}

By the location sensors in the street lamp, the server can be informed whether the street lamp is stolen. Moreover, the street lamp can be found when the street lamp is lost. Further, when the server finds the light bulbs of street lamps are broken, the server can send the detail location to the serviceman for repairing, so the serviceman can locate the broken street lamp accurately which improves efficiency.

\section{Manage Power Adjustment}

In intelligent sensing street lamp, the brightness of street lamp can be adjusted with flexible management platform, the management platform can optimize resource scheduling for easy and highly automated management. The street lamp is equipped with some sensors, such as location sensor, infrared sensor, and light sensor, to form an intelligent sensing street lamp. Consequently, brightness of street lamps can be adjusted.

The infrared sensor in street lamp makes the street lamp more intelligence. The street lamps can distinguish the demands for brightness. Namely, for street lamps in the unmanned area, the brightness should be turn down, and for the street lamps in the crowded area, the brightness needs to be turned up. Therefore, safety in the crowded areas can be guaranteed, and turning down of unmanned street lamps meets energy conservation requirement.

\section{Auto Abnormal Alert}

The abnormal states are:

1. The street lamp bulb is broken. When server checks the street lamp and finds its current is equal to zero but its voltage differs from zero, it concludes the street lamp has a broken bulb.

2. The street lamp is offline. When server checks the street lamp and finds that no data are received from the lamp, it concludes the street lamp is in offline state.

3. The street lamp is in the power saving mode. When server checks the street lamp and finds both its current and voltage are lower than their normal values, it concludes the street lamp is in the power saving mode.

4. Fault. When server checks the street lamp and finds that both lamp current and voltage are equal to zero, and all street lamps in that region have the same state, the server concludes that a fault occurred, which may be caused by a power failure.

5. Close. When server checks the street lamp and finds that both lamp current and voltage are equal to zero, but not all street lamps in that region have the same state, the server concludes that the street lamp is closed.

Autonomous alarm is to avoid stealing. Every street lamp needs to have a self-protection ability. When it is stolen, it should autonomously send the alarm. In this way, the street lamp stealing can be avoided. A flexible management platform is implemented, and it notifies the managers about broken street lamps at real time and automatically dispatches the maintenance staff to repair broken street lamps.

\section{CONCLUSIONS}

In order to satisfy the requirements of smart cities, this paper proposes a smart street lamp (SSL) based on fog computing. The SSL mainly consists of three parts: intelligent sensing street lamp (street lamp brightness can be adjusted and autonomous alarm notifies about lamp abnormal state); efficient network (real-time communication is achieved, the NBIoT is adopted for communication between server and massive street lamps, and the Internet communication 
technology, such as $\mathrm{Wi}-\mathrm{Fi}$ and $4 \mathrm{G}$, is adopted for communication between server and managers); and flexible management platform (management platform can optimize resource scheduling for easy and highly automated management of street lamp system). The proposed SSL was verified by its application in Xiasha district of Hangzhou, China, and obtained results proved high efficiency. The average maintenance period, which denoted the time between the abnormal lamp state appeared and the server checked it, was about 20 minutes. Moreover, the proposed SSL can reduce human resources by eliminating unnecessary periodic inspections.

\section{ABOUT THE AUTHORS}

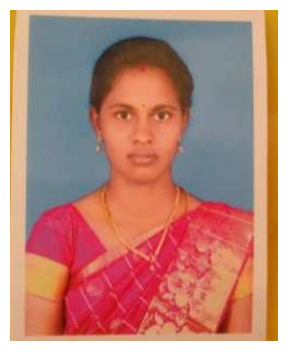

\section{S. ARIVUKODI}

S. Arivukodi received her BSc Degree in Computer Science, MCA Degree in Computer Applications from Bharathidhasan University, Trichy India in the year 2016 and 2019.

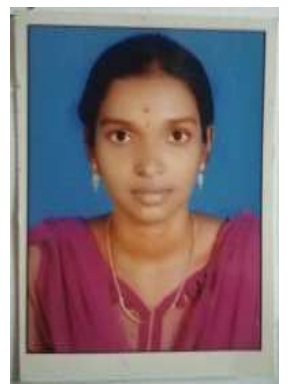

\section{S. BHUVANESHWARI}

S. Bhuvaneshwari received her BCA Degree in Computer Application, MCA Degree in Computer Applications from Bharathidhasan University, Trichy India in the year 2016 and 2019.

\section{REFERENCES}

1. B Chatschik. An overview of the Bluetooth wireless technology. IEEE Communications Magazine, 39(12): 86-94, 2001 .

2. $\quad H$ Farahani. ZigBee and IEEE 802.15.4 Protocol Layers C ZigBee Wireless Networks and Transceivers. Zigbee Wireless Networks \& Transceivers, pp. 33-135, 2008.

3. S Schwarz, C Mehlfuhrer, M Rupp. Calculation of the spatial preprocessing and link adaption feedback for $3 G P P$ UMTS/LTE. Wireless Advanced, pp. 1-6, 2010.

4. R. He, B. Ai, G. Wang, K. Guan, Z. Zhong. High-Speed Railway Communications: From GSM-R to LTE-R. IEEE Vehicular Technology Magazine, 11(3): 49-58, 2016.

5. $\quad$ RS Sinha, Y. Wei, SH Hwang. A survey on LPWA technology: LoRa and NB-IoT. lct Express, 3(1), 2017. 